\title{
Waveform Control Pulsed Field Magnetization of RE-Ba-Cu-O Bulk Superconducting Rings
}

\author{
Y. Tsui, D.A. Moseley, A.R. Dennis, Y.-H. Shi, M.R. Beck, V. Cientanni, \\ D.A. Cardwell, J.H. Durrell, and M.D. Ainslie, Senior Member, IEEE
}

\begin{abstract}
One of the potential applications of ring-shaped, single grain RE-Ba-Cu-O bulk superconductors is in desktop magnetic resonance imaging (MRI) and nuclear magnetic resonance (NMR) systems as an alternative to conventional permanent magnets. The higher magnetic field available from magnetized bulk superconductors could significantly improve the performance of such systems, as well as reduce their size and increase portability. The pulsed field magnetization (PFM) method provides a fast, compact and cost-effective method for magnetizing these materials as trapped field magnets. However, bulk superconducting rings are very susceptible to thermomagnetic instabilities during the PFM process, and thus, to date, the reported trapped fields in ring bulks magnetized by PFM are less than $0.35 \mathrm{~T}$ at the centre of single rings.

In this work, we demonstrate that the trapped field in a superconducting ring bulk can be enhanced significantly by optimizing the waveform of the magnetizing pulse used in the PFM method. This optimization can be achieved easily by using an Insulated Gate Bipolar Transistor as a fast-switching device with a controllable switching frequency in the pulse-generating electric circuit. Our findings represent a key step forward in utilizing bulk, singlegrain superconducting rings magnetized by PFM in portable magnet systems.
\end{abstract}

Index Terms - High-temperature superconductivity, bulk superconductors, bulk superconducting rings, trapped field magnets, pulsed field magnetization, waveform control

\section{INTRODUCTION}

$\mathbf{S}$ TUDIES have been conducted to explore the possibility of exploiting the high field trapping capability of RE-Ba-Cu$\mathrm{O}$ (where $\mathrm{RE}=$ rare earth or $\mathrm{Y}$ ) bulk superconducting rings as an alternative to conventional permanent magnets in desktop NMR and MRI systems to significantly improve their performance, as well as reduce their size and increase portability. Some success has already been demonstrated using RE-Ba$\mathrm{Cu}-\mathrm{O}$ bulk superconducting rings magnetized by the fieldcooled (FC) magnetization method [1,2,3]. For the FC method, large and expensive superconducting magnets and long

This work was supported by the Engineering and Physical Sciences Research Council (EPSRC) Early Career Fellowship, EP/P020313/1 and by EPSRC grant EP/T014679/1. (Corresponding author: Yeekin Tsui.)

Y. Tsui, A.R. Dennis, Y.-H. Shi, M.R. Beck, V. Cientanni, D.A. Cardwell, J.H. Durrell, and M.D. Ainslie are with the Bulk Superconductivity Group, Department of Engineering, Cambridge CB2 1PZ, UK (e-mail: ykt27@cam.ac.uk).

D.A. Moseley is with Robinson Research Institute, Victoria University of Wellington, PO BOX 33436, Petone, Lower Hutt 5046, New Zealand.

Color versions of one or more of the figures in this paper are available online at http://ieeexplore.ieee.org.

Digital Object Identifier will be inserted here upon acceptance. magnetization times (e.g., hours) are required. Alternatively, the pulsed field magnetization (PFM) method provides a fast, compact and cost-effective mean of magnetizing RE-Ba-Cu-O bulk superconducting discs with high trapped fields $[4,5]$. However, the final trapped fields obtained by PFM are generally lower than those obtained using the FC method due to heating during the PFM process associated with the rapid movement of magnetic flux [6]. The heating issue is more serious for bulk superconducting rings. The heat capacity of bulk superconducting rings is smaller than that of the discs with the same outer diameter because of the missing material in the bore. Magnetic flux penetrates into a ring bulk superconductor during the PFM process from both the inner and outer edges of the sample [7,8], which may also lead to higher heating when these flux fronts meet [9], in comparison with disc-shaped superconductors of the same outer diameter. As a result, bulk superconducting rings are more susceptible to thermomagnetic instabilities during the PFM process, making it very challenging to magnetize samples of this geometry to high trapped fields. Thus, in the literature to date, reported trapped fields in bulk superconducting rings magnetized by PFM are less than $0.35 \mathrm{~T}$ at the centre of a single ring bulk compared to those magnetized by FC $[10,11,12,13]$.

The trapped field in a superconducting $\mathrm{RE}-\mathrm{Ba}-\mathrm{Cu}-\mathrm{O}$ disc bulk can be enhanced by controlling the waveform of the magnetizing pulse used in the PFM method [14]. The waveform of the magnetizing pulses can be modified by changing the values of the capacitance, inductance or resistance of the pulse-generating circuit [15]. Alternatively, Hirano et al. [13] increased the rise time of the magnetizing pulses by inserting copper plates into a stack of ring-shaped $\mathrm{MgB}_{2}$ bulk superconductors. In a different approach, Ida et al. [14, 16] used the waveform control pulse magnetization (WCPM) method to modify the waveform of the magnetizing pulses. In this WCPM method, an Insulated Gate Bipolar Transistor (IGBT) was used, which acts as a high-speed switch. The waveform of the magnetizing pulse is modified by changing the switching frequency of the IGBT.

In this work, we have employed the WCPM method (without feedback) to perform single-pulse measurements on a single-grain $\mathrm{Ag}$-containing $\mathrm{Gd}-\mathrm{Ba}-\mathrm{Cu}-\mathrm{O}$ ring bulk superconductor. The measurements were performed at temperatures down to $70 \mathrm{~K}$ using magnetizing pulses with different waveforms, with the rise time and the full-width-half-maximum of the 
magnetizing pulses varied from 25 to $116 \mathrm{~ms}$ and 62 to 245 ms, respectively.

\section{EXPERIMENTAL DETAILS}

\section{A. Sample Details}

The sample studied in this work is a single-grain, annular bulk Ag-containing $\mathrm{Gd}-\mathrm{Ba}-\mathrm{Cu}-\mathrm{O}$ superconductor, fabricated by the top seeded melt-growth technique (TSMG) using the buffer-seed technique [17]. The ring was grown as such, rather than being machined post-growth: the single crystal structure was achieved using a flat circular buffer pellet placed on top of the whole ring, on which the seed crystal was placed. After growth the buffer was removed. The outer diameter, inner diameter and the height of the ring bulk in its final, as-fabricated form were $30.8 \mathrm{~mm}, 12.5 \mathrm{~mm}$ and $11.9 \mathrm{~mm}$, respectively. A photograph of the sample is shown in Figure 1.

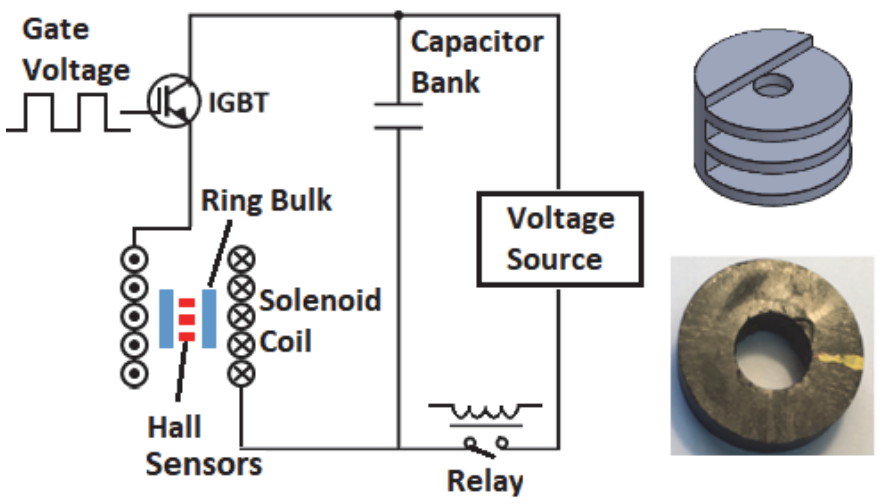

Fig. 1. (Left) A schematic diagram of the pulse generating circuit. (Upper right) A drawing of the copper housing on which the three Hall sensors were mounted. (Lower right) A photograph of the Ag-containing $\mathrm{Gd}-\mathrm{Ba}-\mathrm{Cu}-\mathrm{O}$ ring bulk superconductor studied in this work.

\section{B. Pulsed Field Magnetization System}

The PFM system can generate a pulsed magnetic field with a peak value up to $\sim 5 \mathrm{~T}$. The pulsed field is generated by discharging a capacitor bank (max. voltage $350 \mathrm{~V}$ ) through a copper-wound solenoid or split coil. A schematic diagram of the electric circuit is shown in Figure 1. The IGBT in the circuit serves as a fast-switching device to control how the current flows through the excitation coil when the capacitor is discharging. The IGBT is closed when the gate voltage is in the high state and open when the gate voltage is in the low state. Therefore, the shape of the output magnetic pulses can be changed by controlling the duty cycle (DutyC) and the frequency of the gate voltage to the IGBT. When the DutyC and frequency of the gate voltage to the IGBT is equal to $75 \%$ and $1 \mathrm{~Hz}$ then the function of the IGBT is equivalent to a simple on-and-off switch in the circuit and we refer to this as the IGBT's default state for these settings. The output magnetic pulses at $110 \mathrm{~K}$ with a charging voltage of $187 \mathrm{~V}$ are shown in

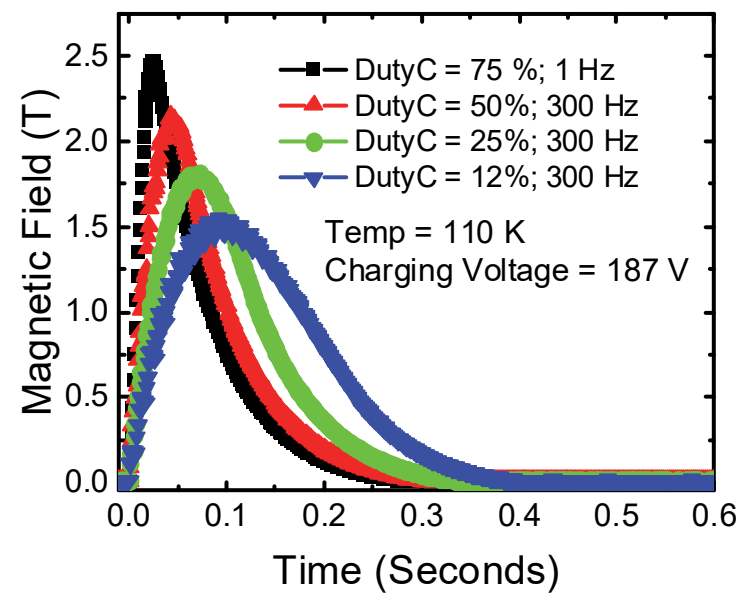

Fig. 2. Applied field versus time at $110 \mathrm{~K}$. The charging voltage was kept the same at $187 \mathrm{~V}$. The rise time of the pulses are $25 \mathrm{~ms}(75 \%$ DutyC and 1 $\mathrm{Hz} \mathrm{SF}), 44 \mathrm{~ms} \mathrm{(50 \%} \mathrm{DutyC} \mathrm{and} 300 \mathrm{~Hz} \mathrm{SF}), 69 \mathrm{~ms}(25 \%$ DutyC and $300 \mathrm{~Hz}$ $\mathrm{SF})$ and $96 \mathrm{~ms}(12 \%$ DutyC and $300 \mathrm{~Hz} \mathrm{SF})$

Figure 2 when different duty cycles and frequencies of the gate voltage were applied. The rise time and the full-widthhalf-maximum (FWHM) of the magnetizing pulses could be modified from 25 to $116 \mathrm{~ms}$ and 62 to $245 \mathrm{~ms}$, respectively. Hereafter, we refer to the peak value of the magnetizing pulse as the 'applied field' for simplicity.

\section{Single-Pulse PFM and Field-Cooling Measurements}

All the single-pulse PFM measurements were performed using a portable PFM system developed by the Cambridge Bulk Superconductivity Group based on a previously reported system for magnetizing disc bulks [5]. The new system uses a Sunpower CryoTel GT cryocooler to provide larger cooling power. Moreover, either a solenoid or split coil can be used for the PFM measurement in the new system. To measure the magnetic field, a linear array of three Hall sensors were mounted in a copper housing, slotted to mitigate any effects from eddy currents induced during the pulse. These sensors are transverse Hall sensors with a sensitivity of 11 to 28 $\mathrm{mV} / \mathrm{kG}$ when an applied current of $1 \mathrm{~mA}$ is used. The distance between two adjacent Hall sensors was $3 \mathrm{~mm}$. The copper housing was then inserted into the bore of the ring bulk such that the Hall sensors were positioned along the crystallographic $c$-axis of the sample. One Hall sensor was at the centre of the bore and the other two were $3 \mathrm{~mm}$ above and below the centre. The copper housing also provided additional cooling to the inner surface of the sample. The sample was first cooled down from the normal state to the superconducting state then a pulsed field was applied to it. Measurements of the trapped field inside the bore of the sample were taken every $0.5 \mathrm{~ms}$ for the first 10 seconds after applying each magnetic pulse and the sampling rate dropped to $100 \mathrm{~ms}$ per data point for the rest of the measurement. The values of trapped field presented in this paper are the values recorded at 15 seconds after applying each pulsed field. Hereafter, we refer to the trapped field at the 
centre of the ring bore as the 'trapped field' in short when the PFM data are discussed.

For the FC measurements, the sample was cooled from room temperature to $77 \mathrm{~K}$, in an applied field of $1.5 \mathrm{~T}$ from a copper electromagnet, using a liquid nitrogen bath. The applied field was then removed and the trapped field profiles at $1.5 \mathrm{~mm}$ above the top and bottom surfaces of the sample were measured using a rotating array of 18 Hall sensors. A handheld gaussmeter was also used to probe the maximum trapped field inside the bore of the sample.

\section{RESULTS AND DISCUSSION}

As shown in Figure 3 the FC measurements at $77 \mathrm{~K}$ show that the trapped field of the sample was quite uniform in the region above the bore of the sample, and the trapped field on the top and bottom surfaces was largely the same, indicating a well-grown, high-quality sample. The maximum trapped field was measured to be $0.65 \mathrm{~T}$ inside the bore of the sample.
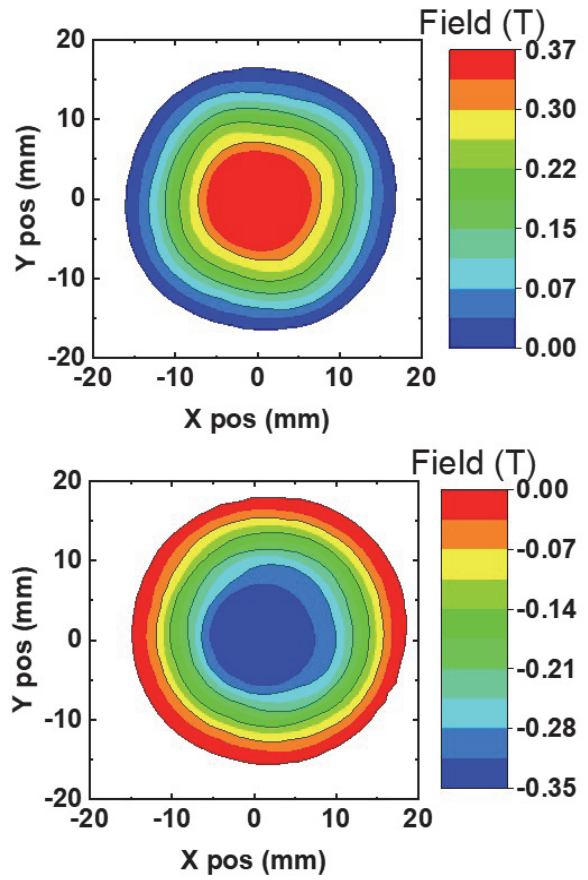

Fig. 3. The trapped field distribution of the sample at $77 \mathrm{~K}$ using the $\mathrm{FC}$ method. Data were taken at $1.5 \mathrm{~mm}$ above the top surface (top) and the bottom surface (bottom) of the sample.

At $77 \mathrm{~K}$ the maximum trapped field obtained by singlepulse PFM when the IGBT was in its default state was $0.58 \mathrm{~T}$ which is already $89 \%$ of the $\mathrm{FC}$ value $(0.65 \mathrm{~T})$. The trapped field increased further to $0.61 \mathrm{~T}$ or $94 \%$ of the $\mathrm{FC}$ value using a $12 \%$ DutyC and $300 \mathrm{~Hz}$ switching frequency (SF). When the IGBT was in the default state, the ring bulk achieved the highest trapped field using the single-pulse PFM method at $73 \mathrm{~K}$.

In order to better understand the effect of the waveform of the magnetizing pulse on the final trapped field, we performed more extensive single-pulse PFM measurements on our sample at $73 \mathrm{~K}$. In Figure 4, we plot the trapped field of the sample against the charging voltage applied to the capacitor bank at $73 \mathrm{~K}$ for different waveforms of the applied magnetizing pulses. When the IGBT was in the default state the maximum trapped field was $0.60 \mathrm{~T}$. The value of the maximum trapped field increased to $0.76 \mathrm{~T}$ using a $25 \%$ DutyC and $300 \mathrm{~Hz}$ SF. At the same time the charging voltage was increased from 187 to $250 \mathrm{~V}$ to achieve the same peak applied field value $(2.4 \mathrm{~T})$. It can be clearly seen in Figure 5 that the wider magnetizing pulse led to a higher trapped field for the same applied field. This also indicates that the enhancement of the trapped field required higher input energy to the capacitor bank. The increase in maximum trapped field saturated as the width of the magnetizing pulse increased further. It might suggest $0.76 \mathrm{~T}$ is very close to the maximum field the sample could trap at 73 K.

At lower temperatures a higher applied field is needed to push flux into a bulk superconductor because of the higher critical current density, which leads to larger heating in the bulk during PFM. Therefore, the maximum trapped field in a bulk sample sometimes does not increase, and can even de-

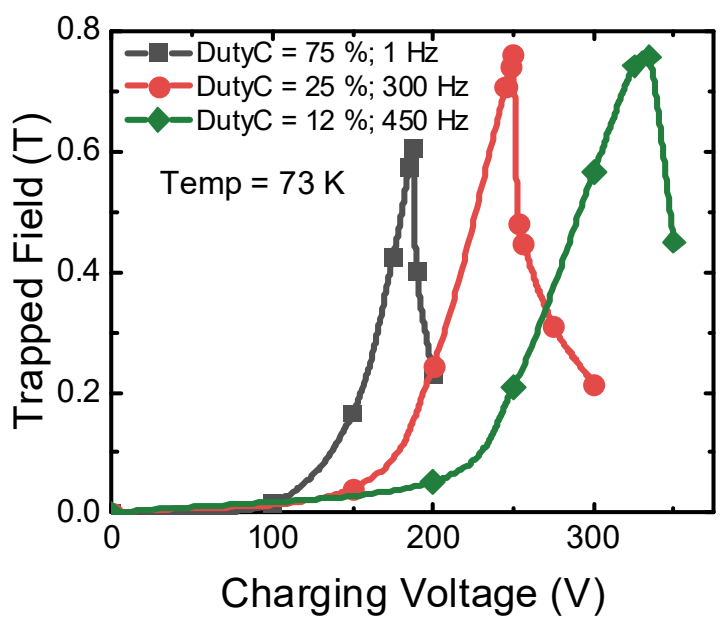

Fig. 4. Trapped field versus charging voltage at $73 \mathrm{~K}$ using magnetizing pulses with different waveforms. The rise time of the pulses varied from 25 ms (75\% DutyC and $1 \mathrm{~Hz} \mathrm{SF})$ to $116 \mathrm{~ms}(12 \%$ DutyC and $450 \mathrm{~Hz} \mathrm{SF})$.

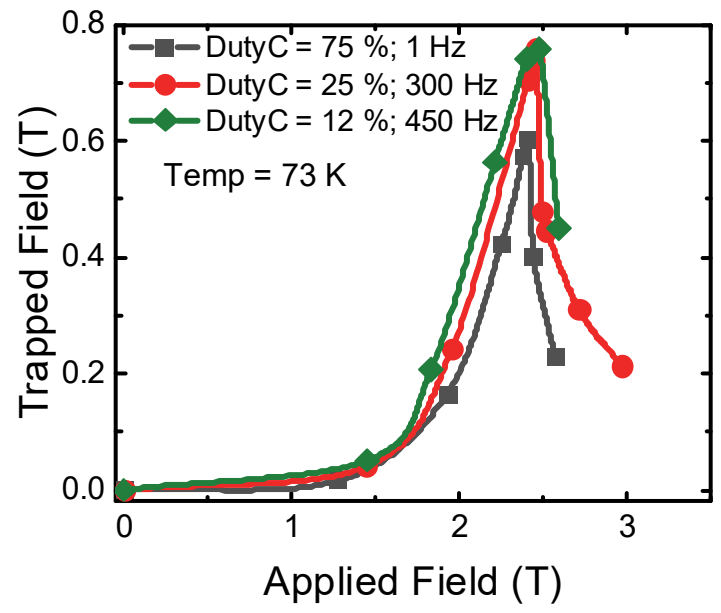

Fig. 5. Trapped field versus applied field at $73 \mathrm{~K}$. Magnetizing pulses with different waveforms were applied. The rise time of the pulses varied from 25 ms (75\% DutyC and $1 \mathrm{~Hz} \mathrm{SF})$ to $116 \mathrm{~ms}(12 \%$ DutyC and $450 \mathrm{~Hz} \mathrm{SF})$. 


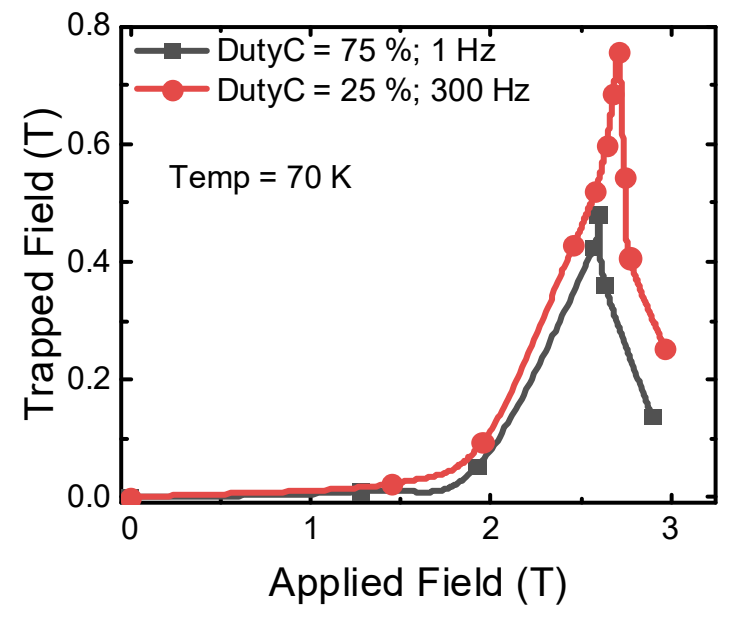

Fig. 6. Trapped field versus applied field at $70 \mathrm{~K}$ using magnetizing pulses of different waveforms. Pulses with a rise time of $25 \mathrm{~ms}$ (75\% DutyC and 1 $\mathrm{Hz} \mathrm{SF})$ or $65 \mathrm{~ms}(25 \%$ DutyC and $300 \mathrm{~Hz} \mathrm{SF})$ were applied.

crease, as the temperature is lowered. As shown in Figure 6, the maximum trapped field of the sample decreased from 0.60 to $0.48 \mathrm{~T}$ as the temperature was lowered from 73 to $70 \mathrm{~K}$ when the IGBT was in the default state. However, the maximum trapped field was enhanced by $58 \%$ to $0.76 \mathrm{~T}$ by applying the optimum magnetizing pulse using a $25 \%$ DutyC and $300 \mathrm{~Hz}$ SF.

In Figure 7 we plot the time dependence of the trapped field in the ring bulk at $73 \mathrm{~K}$ after applying magnetizing pulses with different rise times. When the IGBT was in the default state only a small amount of flux could penetrate into the sample for an applied field of $2.40 \mathrm{~T}$. More flux could be forced to penetrate into the sample by increasing the applied field, but this would also increase the amount of heating and might trigger a flux jump, leading to a lower final trapped field (and possibly a negative trapped field if the circulating magnetizing current is broken) $[18,19]$. As shown in Figure 7, when the IGBT was in the default state for an applied field of $2.44 \mathrm{~T}$, the peak field in the bore of the sample was $2.23 \mathrm{~T}$, which is very close to the applied field, suggesting there was very little shielding current in the bulk superconducting ring. This shows that the sample was almost driven to the normal state and the final trapped field was reduced significantly by increasing the applied field a mere $40 \mathrm{mT}$ from 2.40 to $2.44 \mathrm{~T}$. The longer rise time of the magnetizing pulse also allows more flux to penetrate into the sample because of the longer duration of the peak-field region of the pulse. It can be seen in Figure 7 that for wider pulses more flux could penetrate into the sample, without causing a flux jump, and the final trapped fields were enhanced. In [15], Fujishiro et al. suggested that a magnetizing pulse with a longer rise time reduces the flux propagation velocity in a $\mathrm{RE}-\mathrm{Ba}-\mathrm{Cu}-\mathrm{O}$ bulk during the PFM process, and hence, lowers heating in the bulk due to the viscous loss. This is likely the reason why the wider pulses allow more flux to penetrate into the sample, while reducing heating and leading to a higher trapped field.

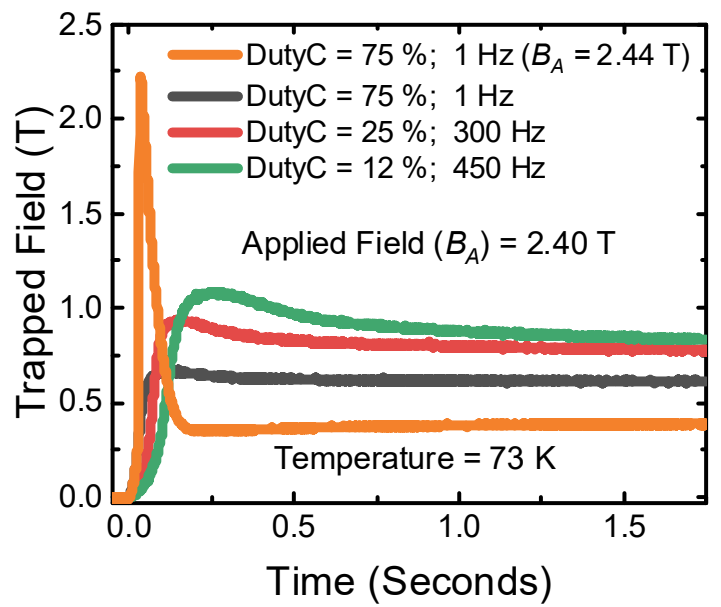

Fig. 7. Trapped field at the sample bore centre as a function of time after applying pulses using magnetizing pulses of different waveforms at $73 \mathrm{~K}$. The rise time of the pulses varied from $25 \mathrm{~ms}$ (75\% DutyC and $1 \mathrm{~Hz} \mathrm{SF})$ to $116 \mathrm{~ms}$ (12\% DutyC and $450 \mathrm{~Hz} \mathrm{SF})$.

\section{CONCLUSION}

We have performed FC and PFM measurements on a single-grain Ag-containing $\mathrm{Gd}-\mathrm{Ba}-\mathrm{Cu}-\mathrm{O}$ ring bulk superconductor at temperatures down to $70 \mathrm{~K}$ using a portable magnetizing system. The magnetic field distribution of the sample obtained by the FC method at $77 \mathrm{~K}$ was quite uniform in the region above the bore and the maximum trapped field was $0.65 \mathrm{~T}$ inside the bore. For the PFM measurements, various magnetizing pulses were applied, with different waveforms generated using an IGBT with controllable switching frequency. With the optimum magnetizing pulses, the trapped fields in the sample were largely increased: i) the trapped field at $77 \mathrm{~K}$ was increased to $94 \%$ of the $\mathrm{FC}$ value using a $12 \%$ DutyC and 300 $\mathrm{Hz}$ SF; ii) at $73 \mathrm{~K}$ the trapped field of $0.76 \mathrm{~T}$ was achieved using a $25 \%$ DutyC and $300 \mathrm{~Hz}$ SF. As far as the authors' knowledge, this is the highest trapped field reported for a RE$\mathrm{Ba}-\mathrm{Cu}-\mathrm{O}$ ring bulk superconductor using PFM to date; iii) at $70 \mathrm{~K}$, the trapped field was increased by $58 \%$ using a $25 \%$ DutyC and $300 \mathrm{~Hz} \mathrm{SF}$. Our findings indicate that, in addition to enhancing the trapped field, the thermomagnetic stability of the PFM process can be greatly improved by modifying and controlling the waveform of the magnetizing pulses.

\section{DATA STATEMENT}

Data related to this publication are available at the University of Cambridge data repository (https://doi.org/10.17863/CAM.75432).

\section{REFERENCES}

[1] T. Nakamura, D. Tamada, Y. Yanagi, Y. Itoh, T. Nemoto, H. Utumi, and K. Kose, "Development of a superconducting bulk magnet for NMR and MRI,” J. Magn. Reson., vol. 259, pp. 68-75, 2015. 
[2] K. Ogawa, T. Nakamura, Y. Terada, K. Kose, and T. Haishi, "Development of a magnetic resonance microscope using a high $T_{\mathrm{c}}$ bulk superconducting magnet," Appl. Phys. Lett., vol. 98, no. 23, 2011, Art. no. 234101.

[3] S. Kim, S. Fukada, R. Nomura, and H. Ueda, "Development of HTS Bulk NMR Relaxometry With Ring-Shaped Iron," IEEE Trans. Appl. Supercond., vol. 28, no. 3, 2018, Art no. 4301505.

[4] H. Fujishiro, T. Tateiwa, A. Fujiwara, T. Oka, and H. Hayashi, "Higher trapped field over $5 \mathrm{~T}$ on HTSC bulk by modified pulse field magnetizing," Physica C, vol. 445-448, pp. 334-338, 2006.

[5] D. Zhou, M. D. Ainslie, Y. Shi, A. R. Dennis, K. Huang, J. R. Hull, D. A. Cardwell, and J. H. Durrell, "A portable magnetic field of $>3 \mathrm{~T}$ generated by the flux jump assisted, pulsed field magnetization of bulk superconductors," Appl. Phys. Lett., vol. 110, 2017, Art. no. 062601.

[6] M. D. Ainslie and H. Fujishiro, "Modelling of bulk superconductor magnetization," Supercond. Sci. Technol., vol. 28, 2015, Art. no. 053002.

[7] E. H. Brandt, "Susceptibility of superconductor disks and rings with and without flux creep," Phys. Rev. B, vol. 55, no. 21, pp. 14513-14526, 1997.

[8] C. Navau, A. Sanchez, E. Pardo, D.-X. Chen, E. Bartolomé, X. Granados, T. Puig, and X. Obradors, "Critical state in finite type-II superconducting rings," Phys. Rev. B, vol. 71, 2005, Art. no. 214507.

[9] M. R. Beck et al., "Dynamics of magnetic flux penetration into ringshaped bulk high temperature superconductors," presented at the $15^{\text {th }}$ European Conference on Applied Superconductivity (EUCAS 2021), 2021.

[10] M. Fabbri, P. G. Albano, M. Pretelli, F. Negrini, and H. Ohsaki, "Flux trapping in a ring-shaped YBCO bulk by pulsed field magnetization," IEEE Trans. Appl. Supercond., vol. 11, no. 4, pp. 4116-4122, 2001.

[11] E. P. Krasnoperov, V. S. Korotkov, and A. A. Kartamyshev, "Magnetization of Superconducting Rings by Long-Duration Pulses," Tech. Phys. Lett., vol. 43, no. 10, pp. 882-884, 2017.

[12] D. Zhou, Y. Shi, A. R. Dennis, D. A. Cardwell, and J. H. Durrell, "Flux jumps in ring-shaped and assembled bulk superconductors during pulsed field magnetization," Supercond. Sci. Technol., vol. 33, no. 3, 2020, Art. no. 034001.

[13] T. Hirano, Y. Takahashi, S. Namba, T. Naito, and H. Fujishiro, "A record-high trapped field of $1.61 \mathrm{~T}$ in $\mathrm{MgB}_{2}$ bulk comprised of copper plates and soft iron yoke cylinder using pulsed-field magnetization," Supercond. Sci. Technol., vol. 33, no. 8, 2020, Art. no. 085002.

[14] T. Ida, Z. Li, M. Miki, M. Watasaki, and M. Izumi, "Waveform Control Pulse Magnetization for HTS Bulk With Flux Jump," IEEE Trans. Appl. Supercond., vol. 28, no. 4, 2018, Art. no. 6801905.

[15] H. Fujishiro, M. Kaneyama, K. Yokyama, T. Oka, and K. Noto, "RiseTime Elongation Effects on Trapped Field and Temperature Rise in Pulse Field Magnetization for High Temperature Superconducting Bulk," Jpn. J. Appl. Phys., vol. 44, no. 7A, pp. 4919-4925, 2005.

[16] T. Ida, S. Watanabe, M. Watasaki, and M. Izumi, "Improvement of trapped magnetic field in GdBCO by waveform control pulse magnetization at $70 \mathrm{~K}$," J. Phys. Conf. Ser., vol. 1559, 2020, Art. no. 012030.

[17] D. A. Cardwell, Y. Shi, and D. K. Namburi, "Reliable single grain growth of (RE)BCO bulk superconductors with enhanced superconducting properties," Supercond. Sci. Technol., vol. 33, no. 2, 2020, Art. no. 024004.

[18] T. Hirano, H. Fujishiro, T. Naito, and M. D. Ainslie, "Numerical simulation of flux jump behavior in $\mathrm{REBaCuO}$ ring bulks with an inhomogeneous $J_{\mathrm{c}}$ profile during pulsed field magnetization," Supercond. Sci. Technol., vol. 33, no. 4, 2020, Art. no. 044003.

[19] P. A. Brazhnik and E. P. Krasnoperov, "Visualization of the Field Penetration at the Shielding Current Break and Its Restoration in the Pulse Magnetized YBCO Ring," J. Supercond. Nov. Magn., vol. 35, p. 1085-1090, 2021. 